\title{
Hyposensibilisierung mit Allergoiden: wirkungsstark und sicher
}

D ie Anforderungen an Allergene für die spezifische Immuntherapie (SCIT) sind seit dem Inkrafttreten der Therapieallergene-Verordnung im Jahr 2008 hoch. Über eine umfassende Evidenz verfügen depigmentierte Allergoide. Der Nachweis der klinischen Wirksamkeit und der Sicherheit sei in mehreren Studien erbracht worden, einschließlich aktueller Phase-III-Studien mit höchsten wissenschaftlichen Standards, berichtete PD Dr. Oliver Pfaar, Wiesbaden. Der primäre Endpunkt der neuen Studien war der kombinierte Symptom-Medikations-Score (SMS), der von der European Medicines Agency als Wirksamkeitskriterium empfohlen wird.

Für die perenniale SCIT mit Baumpollen-Präparaten $\left(\right.$ Depigoid $^{\circledR}$ ) wurde in zwei plazebokontrollierten, randomisierten Doppelblindstudien eine hohe Wirksamkeit sowohl in der ersten als auch in der zweiten Baumpollen-Saison dokumentiert. Der SMS sank um 36,5\% $(\mathrm{p}=0,004)$ bzw. 39,0\% ( $\mathrm{p}=0,01)$ gegenüber Plazebo (Höiby AS et al. Clin Exp Allergy 2010; 40: 1062-70 und Pfaar O et al. Allergy 2010; 65: $1614-$ 21). Eine klinisch relevante Wirksamkeit nach Malling ist gegeben, wenn die SMS-Differenz zwischen der Verumund Plazebogruppe über $30 \%$ beträgt. Die Responderrate lag bei $64 \%$ in der Verumgruppe gegenüber $32 \%$ in der Plazebogruppe ( $\mathrm{p}=0,0008)$. Außerdem kam es zu einem Anstieg der spezifischen IgG4-Antikörper. Somit habe die SCIT neben der klinischen Wirksamkeit auch einen immunologischen
Effekt, betonte Pfaar. Die Sicherheit der SCIT mit den Baumpollen-Präparaten war insgesamt gut; es traten keine Grad-3- und keine Grad-4-Reaktionen auf.

Auch eine Rush-Aufdosierung ist möglich, wie eine Studie zur KurzzeitSCIT mit depigmentierten Allergoiden (Depiquick $^{\circledR}$ ) ergab. Auch hier traten keine Grad-3- oder Grad-4-Reaktionen auf, obschon die Patienten an einem Tag auf die Maximaldosis auftitriert wurden (Brehler R et al. Allergy Asthma Proc 2010; 31: 1-8). Mit einer Kurzzeit-SCIT von nur sieben Injektionen ist nach diesen Daten eine hohe Wirksamkeit zu erreichen. Der kombinierte SMS sank um 39,5\% im Vergleich zu Plazebo $(\mathrm{p}<0,01) . \quad$ Abdol A. Ameri

Symposium „Ära der Evidenz-basierten SIT“ im Rahmen des 6. Deutschen Allergiekongresses. Wiesbaden, 8. September 2011. Veranstalter: Novartis Pharma, Nürnberg

\section{Allergische Rhinitis kosteneffizient behandelt}

D ie allergische Rhinitis wird noch zu selten als Krankheit ernst genommen, die zum Teil schwerwiegende Folge- und Begleiterkrankungen nach sich zieht, warnte PD Dr. Martin Wagenmann, Düsseldorf. Unterschätzt würde u.a. die Beeinträchtigung der geistigen Leistungsfähigkeit durch die Symptomatik. Auch werde nicht ausreichend realisiert, dass allergische Rhinitis ein wesentlicher Risikofaktor für Asthma sei. Trotz der verfügbaren Möglichkeiten bleibe die Behandlung deshalb häufig insuffizient. Wagenmann empfahl, Patienten mit allergischer Rhinitis gezielt nach persistierenden Beschwerden zu befragen und rechtzeitig intranasale Glukokortikoide einzusetzen, wie z. B. Mometason (Nasonex ${ }^{\circledR}$ ).

Das Leitlinien-Update 2008 der Initiative „Allergic Rhinitis and its Impact on Asthma" (ARIA) führt intranasale Glukokortikoide als wirksamste Pharmakotherapie bei allergischer Rhinitis (Bousquet J et al. Allergy 2008; 63 [Suppl 86]: 8-160). Allerdings unter- scheiden sich die Präparate in der Kosteneffizienz, wie die Intercontinental Marketing Services Health (IMS Health) im Jahr 2009 mithilfe ihrer Datenbank IMS ${ }^{\circledR}$ Disease Analyzer ermittelt haben. Bei einer Studienpopulation von Patienten mit allergischer Rhinitis prüften IMS Health den Verbrauch und die daraus resultierenden Kosten

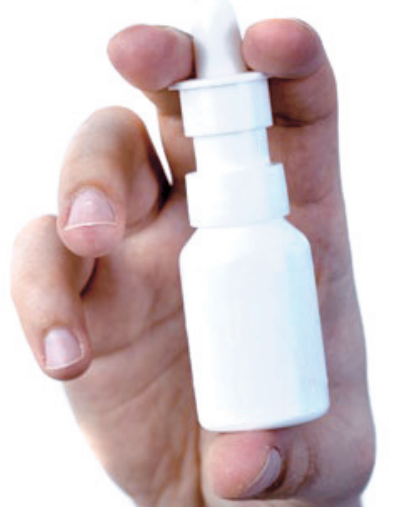

Was wirklich im Nasenspray steckt, zeigt sich im Verbrauch. von Mometason- gegenüber Budenosid-haltigen Nasensprays unter RealLife-Bedingungen, aufgeteilt nach hausärztlich und HNO-ärztlich behandelten Patienten. Die Marktforscher kamen zu dem Ergebnis, dass mit Mometason therapierte Patienten im fraglichen Zeitraum insgesamt deutlich weniger der Studiensubstanz - gemessen an den Hüben - verbrauchten, als Patienten, die Budesonid-haltiges Nasenspray verordnet bekamen.

„Letztendlich entlastete der Einsatz von Mometason das Arzneimittelbudget der Ärzte“, erklärte Prof. Dr. Ludger Klimek, Wiesbaden. Schon auf der Basis der ermittelten Patientenverteilung für „Erstverordnungen“ im MAT 09/08 auf der Basis von Disease Analyzer ergebe sich eine Kostenersparnis in Höhe von 2.387.793 Euro für die GKV. Die Ersparnis bei Einbeziehung der gesamten Patientenpopulation dürfte weit höher ausfallen.

Ute Ayazpoor

Symposium „Die wirtschaftliche Therapie der allergischen Rhinitis in der Praxis“ im Rahmen des 6. Deutschen Allergiekongresses. Wiesbaden, 8 . September 2011. Veranstalter: MSD, Haar 